\title{
Praxis Lúdica Docente en la Enseñanza de las Ciencias Sociales. Una Visión desde la Didáctica Diferenciada
}

\section{Playful Educational Praxis in the Teaching of Social Sciences. A View from Differentiated Didactics}

\author{
Zoila Rosa Montiel-Florez \\ zoyimona@gmail.com \\ Institución Educativa San Marquito, Departamento de Sucre \\ Colombia \\ https://orcid.org/0000-0003-3465-1699
}

Recibido: 15 de noviembre de 2019

Aprobado: 16 de diciembre de 2019

\begin{abstract}
RESUMEN
El estudio que se desarrollará tiene como propósito principal describir la praxis Lúdica docente en la enseñanza de las Ciencias Sociales como una visión desde la didáctica diferenciada. Es una investigación de naturaleza cualitativa, a fin de comprender a los actores sociales, además de la investigadora quien se involucra de manera objetiva y fiable en el proceso investigativo, orientada por una metodología descriptiva, la información se obtendrá mediante la observación y las entrevistas semi estructuradas a 04 sujetos, a fin de obtener diferentes perspectivas sobre el fenómeno. En este sentido, este artículo desglosa la importancia de la utilización de los ejercicios recreativos para la mejora de diferentes ideas y el impacto que tiene la praxis lúdica docente, ya que proporciona actividades, donde el educador potencia la enseñanza aprendizaje para avanzar en el proceso de formación convirtiéndose en un dispositivo clave para que el niño se familiarice con el aprendizaje significativo en condiciones agradables de una manera seductora creando habilidades desarrolladas mediante la ejecución de juegos.
\end{abstract}

Descriptores: Praxis Lúdica; docente; enseñanza; ciencias sociales; didáctica diferenciada.

\section{ABSTRACT}

The study that will be carried out has the main purpose of describing the playful teaching practice in the teaching of Social Sciences as a vision from the differentiated teaching. It is a qualitative research, in order to understand the social actors, in addition 


\section{Zoila Rosa Montiel Florez}

to the researcher who is objectively and reliably involved in the research process, guided by a descriptive methodology, the information will be obtained through observation and semi-interviewing. structured to 04 subjects, in order to obtain different perspectives on the phenomenon. In this sense, this article breaks down the importance of the use of recreational exercises to improve different ideas and the impact that playful teaching praxis has, since it provides activities, where the educator promotes teaching - learning to advance the process. of training becoming a key device for the child to become familiar with meaningful learning in pleasant conditions in a seductive way creating skills developed by playing games.

Descriptors: Playful Praxis; teacher; teaching; social sciences; differentiated teaching.

\section{INTRODUCCIÓN}

En el contexto educativo, la lúdica se percibe como una alternativa vital a nivel pedagógico, generando satisfacción y estímulo en su ejecución, además permite incluir temas concernientes a las diferentes áreas, disciplinas y lo más importante es la inclusión de los valores tanto personales como los relacionados con el proceso educativo.

En ese sentido, el ser humano a lo largo de su historia se ha visto abocado a hacer del aprendizaje una constante en su vida, para solucionar los desafíos que se le presentan o como búsqueda de solución a los propios. Es así como el aprender se presenta como una actividad de descubrimiento, encuentro de sí y del ambiente que rodea al individuo, situación que a nivel social es organizada por las instituciones, quienes por medio del currículo y las normas educativas lo convierten en una forma de integración, socialización y endoculturación.

En esta dupla aprendizaje-educación es pertinente buscar las formas más efectivas y agradables que generen gusto y una actitud activa e integradora de trabajo en equipo, que se aleje de actividades educativas alienantes, aburridoras para transformarse en espacios motivadores del aprendizaje. Dichas actividades deben responder a la vida y a valores positivos como el bienestar, la felicidad y el dinamismo, articulables a través de la acción lúdica con compromiso, interacción y conciencia. Así mismo, deben estar en la búsqueda de comprender e incluir desde todas las perspectivas probables, 


\section{Zoila Rosa Montiel Florez}

no solamente desde lo cognitivo, y en este sentido abarcar la multidimensionalidad del ser humano.

Asimismo, cada nación requiere ajustar la educación a su entorno cultural en búsqueda de respuestas acordes a sus propias circunstancias sociales, para lo cual deben adaptarse a la sociedad formando seres reflexivos, con gran capacidad para resolver problemas, aprender de modo autónomo, que puedan asimilar los contenidos de la cultura a la que pertenecen, apropiándose de aquellos modos de pensar, sentir y hacer, los cuales garanticen una interacción inteligente en contextos cambiantes, complejos, así como la participación responsable, creativa y transformadora de la educación replanteándose objetivos, metas, pedagogía, didácticas, si quiere cumplir con su misión en la sociedad, pues la educación es tarea de humanización.

En el mismo orden de ideas puede señalarse que la enseñanza y el aprendizaje de las ciencias sociales es un tema recurrente y polémico en el debate educativo actual; no solo por los conocimientos que se comunican, sino por los valores que a través de su formación se inculcan en la conciencia y en la práctica de los sujetos educandos. La naturaleza de las disciplinas sociales y sus orientaciones constituyen un soporte para despertar el interés por el desarrollo humano, el bienestar social y la preservación del planeta.

En estos procesos didácticos innovadores y transformadores de la sociedad actual, las estrategias didácticas son las herramientas básicas para que las generaciones actuales transformen en conocimiento, esa cantidad de información que les llega de las diferentes fuentes de información, de allí entonces la razón de abordar la lúdica desde la perspectiva de las ciencias sociales, buscando con ello el engranaje necesario para la consolidación del aprendizaje efectivo.

\section{DESARROLLO TEÓRICO}

\section{La Lúdica y su significatividad en los contextos educativos}

Según Jiménez (2012, P. 42), la lúdica es una dimensión del desarrollo humano que fomenta el desarrollo psicosocial, la adquisición de saberes, la conformación de la personalidad, encierra una gama de actividades donde se cruza el placer, el goce, la actividad creativa y el conocimiento, considerándose más como una condición, una 


\section{Zoila Rosa Montiel Florez}

predisposición del ser frente a la vida, frente a la cotidianidad y el convivir diariamente e interrelacionarse con su entorno socio-educativo como familiar-ambiental.

Es una forma de estar en la vida, de relacionarse con ella en esos espacios cotidianos donde se produce disfrute, goce, acompañado de la distensión de las actividades simbólicas e imaginarias con el juego, la chanza, el sentido del humor, el arte y otra serie de actividades producidas cuando se interactúa con otros, sin más recompensa que la gratitud que producen dichos eventos, es una manera de vivir la cotidianidad, sentir placer y valorar lo que acontece percibiéndolo como acto de satisfacción física, espiritual o mental que siente la persona cuando está en plena satisfacción interacción.

Así también para Motta (2014), la lúdica es un procedimiento pedagógico en sí mismo, la metodología lúdica existe antes de saber que el profesor la va a propiciar genera espacios y tiempos, provoca interacciones y las situaciones lúdicas se caracterizan. Ello se interpreta, como un medio que resulta en la satisfacción personal a través del compartir con los otros, en la interrelación que nace entre los seres que se sienten satisfechos con su medioambiente y regocijados con su ser.

En el mismo orden de ideas, la lúdica, para Díaz (2006), la lúdica son actividades comunes dentro del proceso de enseñanza-aprendizaje, buscando mejorar el rendimiento de los estudiantes, por ello, su aplicación es común en la educación inicial por cuanto genera desarrollo de pensamiento creativo, apoya los procesos de aprendizaje y modela procesos didácticos alternativos a los tradicionales al generar motivación intelectual, así como favorecer la relación maestro-estudiante, por cuanto el aprendizaje no sólo es un proceso cognitivo, sino también es un proceso socio afectivo, por ser esta la esencia de la lúdica el establecimiento de afectividad y emocionalidad entre los integrantes.

En el contexto de la educación las actividades lúdicas deben ser incorporadas permanentemente en el aula por ser una fuente inagotable de aprendizaje donde el alumno, aprende jugando divirtiéndose con lo aprendido, esto hace que su aprendizaje no sea tedioso ni rígido, viven la cotidianidad, desarrollan habilidades, destrezas, actitudes acordes con su comportamiento cognitivo y crecimiento intelectual, relacionando lo que aprende jugando con los actos de su vida. 


\section{Zoila Rosa Montiel Florez}

\section{Cómo funciona la creatividad en las Actividades Lúdicas}

De acuerdo con el criterio de Matos (2012), la actividad lúdica, es un factor de evolución de la personalidad en el mismo existen varios tipos de actividades lúdicas creativas entre los cuales se encuentran las corporales y las socias afectivas, como los juegos de actitudes o corporales; los cuales son actividades que realiza el individuo y generan un desgaste de energía que lo impulsa a generar acciones creativas divertidas, afectivas entre otras.

También están los juegos pasivos; los cuales se pueden definir como todas aquellas actividades consideradas diversiones o socio afectivas, en las cuales los niños y niñas invierten un mínimo de energía, producen contribuciones importantes para las buenas adaptaciones personales, sociales de ellos, le permite relajarse cuando están solos, fomentan la socialización en actividades grupales a todas las edades, los educandos se dedican tanto a los juegos activos como pasivos y el tiempo que le dedican a cada uno depende de la salud de los mismos, del placer que le proporciona, del momento en el que aprenden los juegos y del interés, que en ellos despierte.

En tal sentido, Montenegro (2010), considera a las actividades lúdicas en el aula como estrategia que permite estimular de manera determinante el desarrollo del niño y niña, debido que a través de estas actividades se pueden fortalecer los valores, estimular la integración, reforzar aprendizaje, promover el seguimiento de instrucciones, el desarrollo psíquico, físico y motor, estimular la creatividad, además de ofrecer igualdad de oportunidades y condiciones para la participación del infante como parte de un grupo diferente al familiar lo cual con lleva a la formación ciudadana.

A través de la expresión lúdica, los niños desde su edad preescolar pueden ejercitar la observación, asociación de ideas, así como la expresión oral; facilitándole de esta forma la adquisición de conocimientos previstos en la planificación y ejecución de proyectos de aprendizaje acordes a su nivel educativo y las expectativas que se tienen en esta edad inicial en relación al aprendizaje significativo de los educandos.

Resulta entonces, de relevante importancia para el docente de educación asuma con verdadera responsabilidad su papel en la formación del alumno dándole la oportunidad de satisfacer sus necesidades e intereses a través de experiencias, situaciones y actividades que le permitan su desarrollo integral, lo preparen para vivir 


\section{Zoila Rosa Montiel Florez}

en sociedad, estimulen sus habilidades y potencialidades.

Evidentemente, que la actividad lúdica está contemplada como un medio para dar cumplimento a uno de los fines educativo, de allí que el docente, está obligado a proveer a los alumnos a actividades que desarrollen en ellos la naturaleza cognitiva, el desarrollo psicomotriz, la lingüística y toda acción social que sea favorable a su crecimiento e interacción inter personal con el entorno social, familiar y comunal.

En el contexto de la educación, específicamente en el área de las ciencias sociales obviamente que la puesta en práctica de este tipo de actividades lúdicas, requiere de la buena intención del docente, la adecuada preparación y el fortalecimiento de sus saberes pedagógicos, lo cual se convierte en una vía expedita hacia la consecución exitosa de los objetivos planteados, ello contempla un real y claro conocimiento para tratar en la medida de lo posible de ofrecer a los alumnos situaciones reales que le sean familiares y le resulten atractivas para aprenderla.

\section{Estrategias de Aplicación de la lúdica en los contextos educativos}

La estrategia de aplicación, es un conjunto de actividades por medio de las cuales se orienta una persona hacia el logro de un objetivo, de acuerdo con lo planteado por Zambrano (2007), quien agrega, además, es un ejercicio donde estudiante y docente logran el éxito en la comunicación, en el despliegue pleno de potencialidades de todos, permitiendo inferir que la experiencia educativa se convierte en una actividad placentera, enriquecedora y ágil, como una herramienta de mejorar la calidad de vida, la independencia personal, obteniendo un desarrollo armónico acorde con las exigencias del medio donde se desarrolló.

Así mismo, Gallego (2012), considera que las estrategias de aplicación son un conjunto de acciones realizadas por el docente con una intencionalidad pedagógica clara y explícita, en estas estructuras de actividad, en las que se hacen reales los objetivos y los contenidos, se componen por el estilo de enseñanza; el tipo de estructura comunicativa, como parte de la cultura escolar, de las relaciones interpersonales.

En ese sentido, las estrategias de aplicación en las actividades lúdicas el compromiso, la responsabilidad y cooperación fungirán como pilares fundamentales para la correcta 


\section{Zoila Rosa Montiel Florez}

aplicación de dicha actividad que es una estrategia activa que desencadena la potencialidad del estudiante por medio de la estimulación y ejercitación para su desarrollo físico e intelectual acorde a su edad cronológica, así como la forma de organizarla, también brinda una gama de motivaciones, relaciona el nuevo aprendizaje con la conducta que el educando ya posee, analiza los objetivos propuestos, anuncia el nuevo aprendizaje y elabora un plan de trabajo acorde a las necesidades. Igualmente, Alvarado (2010), define a la estrategia de aplicación como las acciones que permiten la participación activa del docente en el desarrollo psico social, motriz, intelectual, así como la personalidad, mediante herramientas lúdicas que tiene como propósito estimular el desarrollo y mantenimiento del movimiento humano, la capacidad mental y aspectos de tipo emocional del participante, reforzando así las áreas principales en las que presentan mayor dificultad.

Visto, así como lo plantea el autor, grande son los beneficios que adquieren los estudiantes, mediante la actividad corporal, el piensa, aprende, crea y afronta los problemas, desarrolla la actividad motriz, considerándose el punto de partida del desarrollo de la inteligencia del estudiante, por cuanto permite reforzar las áreas principales en las que presentan dificultades tanto para relacionarse como para actuar en su medio ambiente.

En relación con la contextualización de las estrategias de aplicación en educación es una necesidad por cuanto apoyan la implementación de ellas en actividades lúdicas creativas de índole corporal, afectiva e intelectual, estimulan el aprendizaje significativo de los niños y niñas con acciones motivadoras que estimulan la creatividad y la adaptación de ellos al medio ambiente, al contexto familiar y las relaciones con los demás.

\section{Juegos Didácticos}

Para Huizinga, citada por Dávila (2013, p.7), el juego "es una acción o actividad voluntaria que se desarrolla sin interés material, realizada dentro de ciertos límites fijos de tiempo y espacio", según una regla libremente consentida pero completamente imperiosa, provista de un fin en sí misma acompañada de un sentimiento de tensión y alegría contagiosa, motivadora, donde el alumno disfruta de su aprendizaje jugando, 


\section{Zoila Rosa Montiel Florez}

por cuanto el juego es esencial para desarrollar músculos, ejercitar todas las partes del cuerpo.

En cuanto al origen de algunos juegos el autor precitado, señala el sonajero, el boliche y el trompo han sido considerados como juegos que se remontan hasta los primitivos, de la misma forma, los esquimales practicaban el juego mágico del boliche sin ninguna significación mágica, mientras la prohibición de cazar al día siguiente constituye testimonio de la persistencia de un tabú cuyo sentido, aún hoy día, se ignora constituyendo estudio de los etnólogos su determinación, de donde se desprende que los juegos permiten a veces sondear la historia ignorada de algunas civilizaciones.

De allí, que Dávila (2013), describe el juego como expresión de las estrategias visuales ya usadas en el siglo XVIII, dando origen a las obras híbridas o Cadavre Exquis de los surrealistas, una versión del juego de salón llamado cabeza, cuerpo y piernas; para su realización, cada artista dibujaba objetos, paisajes y detalles de figuras humanas y animales, en una parte del lienzo o cartón, una vez terminada su parte, se cubría con papel, para que el siguiente pintor no sufriera la influencia de la obra anterior, igual ocurre en el juego cabeza, cuerpo y piernas, cada jugador dibuja en un papel una parte del cuerpo y después se dobla para que el siguiente no vea lo hecho por el anterior.

Por otra parte, para Platón (427-348), citado por Núñez (2010), considera que el juego entre las sociedades más primitivas tenía una caracterización propias de las culturas de esas agrupaciones, se luchaba más por la supervivencia que por la recreación y diversas como placer natural el juego no era una actividad de esparcimiento y relax como lo es actualmente sino competitivo, sin embargo, la educación debía comenzar a los 7 años de edad, donde los primeros años de vida del niño debían estar ocupados en juegos educativos mixtos, bajo vigilancia del adulto en jardines infantiles, preferían darle al juego un valor pedagógico netamente educativo y recreativo más que competitivo, al producir daño en la formación del niño.

En el mismo sentido, Núñez (2010, p. 15), hace referencia en este recuento histórico a Montessori con su creación de la "casa de los niños" en Roma, atiende a la población infantil, de 3 a 6 años, de escasos recursos económico, donde aprenden a ordenar las sensaciones, la integración y adaptación a la escuela específicamente al pre-escolar 


\section{Zoila Rosa Montiel Florez}

dependiendo en gran medida del grado de empatía que el docente pueda trasmitir desde el mismo momento al recibir al educando, separado por primera vez del seno familiar, para enfrentar la experiencia de la vida escolar; esa relación se establece en el nivel de Preescolar esta obviamente marcada por las actividades programada por el educador para "ganar" su atención y despertar curiosidad.

Es importante destacar también, que para establecer una relación empática en el aula de clase entre el docente y el párvulo en el nivel preescolar, deben darse ciertas condiciones tales como: conocimiento por parte del educador de las características psicológicas, físicas y cognitivas del niño o niña, puesta en práctica las actividades que motiven y capten la atención del infante, además de la buena voluntad del docente para llevarlas a cabo.

En este sentido, la mejor forma de promover una adaptación efectiva del educando al preescolar lo representa la utilización de actividades lúdicas que a través de ellas se permite la interacción efectiva del alumno con sus compañeros, docentes así como el afianzamiento de normas valores, aprendizajes significativo, para fortalecer su desarrollo cognoscitivo motriz, social; es decir, su desarrollo integral, tal como lo plantea la Pedagogía y Psicología Infantil (2010, p. 21), al afirmar los juegos en la actualidad son utilizados en las escuelas como elemento educativo,

De allí, que el estudiante, es un ser cargado de emociones, hiperactivo, ansioso de realizar actividades por lo que se hace necesario que el docente tenga a mano herramientas didácticas satisfactorias de actividades lúdicas adecuadas al ubicar al educando en su ambiente natural, generalmente estos requieren de mucha actividad física, especialmente aquellos que se realizan al aire libre, los cuales, resultan ser sus favoritos porque involucran actividades como correr, saltar, tironear y cualquier otro ejercicio corporal que suponga gran empleo de energía vital.

Además, de conocer las características del estudiante, el docente debe estar consciente cuales son las necesidades e intereses de este, de manera que al inducir las actividades lúdicas dentro de la planificación, desarrollo y evaluación de trabajo escolar con el real y claro objetivo de estimular su desarrollo integral, planteamiento que reafirma Zielinski (2010, p. 430), al señalar: "El juego es un móvil de fundamental importancia en el proceso de evolución infantil; desarrolla sus potencialidades por su 


\section{Zoila Rosa Montiel Florez}

ejercitación placentera, espontánea, de expresión de ideas, sentimientos, es elaboración de temores, angustias, es definitiva una actividad creadora".

Obviamente, que la puesta en práctica de este tipo de actividades, requiera de la buena intención del docente, la adecuada preparación y el fortalecimiento de sus saberes pedagógicos, lo cual se convierte en una vía expedita hacia la consecución exitosa de los objetivos planteados, ello contempla un real conocimiento escolar para tratar en la medida de lo posible de ofrecer al educando situaciones reales que le sean familiares y le resulten atractivas.

Del mismo modo, debe el docente tener claro conocimiento cual es el alcance de utilizar actividades lúdicas dentro de la planificación escolar, por cuanto el juego favorece el desarrollo cognoscitivo en muchas áreas, en el juego con el movimiento, el preescolar llega a conocer la velocidad, el peso, la gravedad, la dirección y el equilibrio, se da cuenta que estos tienen usos, propiedades comunes y apropiadas en su juego con otros practica los conceptos y roles sociales, aprendiendo además algunos aspectos de su cultura.

Es importante señalar, que aun con el conocimiento de la importancia que tiene el juego para el niño y niña en edad preescolar y los efectos positivos que este puede causar al utilizarse con fines pedagógicos en este caso particular para estimular el desarrollo integral de los mismos, no se trata de convertir el aula en una "sala de juegos" sino de aprovechar la siempre favorable disposición del niño hacia las actividades lúdicas para orientar, con base en sus necesidades e intereses individuales, el objetivo propuesto.

Para ello, se hace necesario que el docente conozca qué tipo de actividades lúdicas puede desarrollar, al tiempo de también conocer cuáles son aquellas que el educando práctica en su cotidianidad y cuales son propias del contexto donde se encuentra inmersa la escuela, para lo cual debe activarse el óptimo funcionamiento de la triada escuela-familia-comunidad, con la finalidad de establecer un trabajo mancomunado permitiendo la puesta en práctica de un modelo educativo, verdaderamente contextualizado, atractivo, integrador, centrado en el niño, además de asegurar la participación de todos los factores relacionados con su formación para la vida y por ende para su desarrollo integral. 


\section{Zoila Rosa Montiel Florez}

De igual manera, se abre una ventana para consolidar este fin y darle mayor sentido al rol del docente a través de la utilización de las actividades lúdicas donde el juego satisface muchas necesidades en la vida de un estudiante : la necesidad de ser estimulado y divertirse, de expresar su exuberancia natural, de ensayar el cambio por el valor intrínseco de este, de satisfacer su curiosidad, de explora y experimentar en condiciones exentas de riesgo, cumple además muchas funciones útiles en el desarrollo infantil, favorece el crecimiento de las capacidades sensoriales de las habilidades físicas, brindado oportunidades de ejercitar y ampliar las capacidades intelectuales.

\section{Rondas o Dramatizaciones}

De acuerdo con el criterio de Matos (2012), los juegos de dramatización donde además de representar a las personas en la vida real o en los medios de comunicación, se ponen de manifiesto la capacidad de fantasía en la cual los niños adoptan papeles dramáticos, heroicas, fantásticas y alejados de la vida real, Incluso se presentan los amigos imaginarios o relativos exagerados de situaciones vividas por ellos que las disfrutan igualmente si las vivieran en su propia realidad, en esta etapa también imaginan situaciones satisfactorias.

Este tipo de juegos puede ser reproductivo o productivo, se le conoce como creativo, por cuanto estimula su imaginación y su creatividad, la duración de esta etapa varía en cada niño, pero puede ubicarse hasta finales de su vida preescolar. Sin embargo, existe otro tipo de juego el activo que puede ubicarse en esta misma etapa de crecimiento, es el denominado juego constructivo, el cual no es sino alrededor de los 5 a los 6 años de edad, que él utiliza los materiales de forma específica y apropiada para fines establecidos por él mismo en el proceso aprendizaje en el cual está inmerso.

En criterio de Pacheco (2015), también, estimulan la imaginación y la creatividad del niño y niña, una de las actividades que más le gusta son aquellas relacionadas con la música, considerada como un juego activo cuando él interviene cantando, tocando un instrumento o cuando utiliza la música como complemento de otro tipo de juego activo; también puede ser considerada reproductiva, cuando ellos cantan, bailan, repiten las 


\section{Zoila Rosa Montiel Florez}

palabras enseñadas y productivas, cuando inventa sus propias palabras para las canciones conocidas por él, crea su música o nuevos pasos de baile, el juego, es el medio ideal para el aprendizaje a través de él va descubriendo el ambiente que lo rodea además de conocerse así mismo.

Igualmente, Arce (2015), manifiesta que en el área de Aprendizaje a través de la dramatización, el niño desarrolla la función simbólica o capacidad representativa, la cual consiste en la representación de algo presente, aspecto que juega un papel decisivo en su desarrollo integral, esta área está diseñada para facilitar a los niños experiencias de dramatización espontáneas. De allí, que donde el estudiante experimenta cómo se sienten otras personas en sus oficios, hogar y profesión, en cuanto a sus logros, miedos y conflictos, favoreciendo así su desarrollo socioemocional.

\section{Dinámicas de Grupo}

El trabajo en grupo permite adoptar decisiones, llegar a conclusiones más ricas que aquellas que son producto del pensamiento individual, además aumenta la calidad de las decisiones de dirección mediante la experiencia e inteligencia colectiva, disminuye la incertidumbre, el riesgo a equivocarse en esta toma de decisiones. El grupo permite también la creación de hábitos, valores, creencias y el perfeccionamiento de las habilidades para lograr cambios en la conducta y dinámica del grupo, en las relaciones personales, permite intercambiar conocimientos y auto reflexionar sobre su trabajo a las personas que lo integran.

Según, Pacheco (2015), un grupo son dos ó más personas que se encuentran en relación psicológica explícita entre sí, existiendo la necesidad de algún género de acción mutua directa, un grupo, no es una simple colección de individuos, sino una interrelación de personas con un objetivo común, desde esa óptica, un grupo es un conjunto de componentes (miembros), cuya interacción engendra nuevas cualidades, no poseen elementos integrantes por separado, cabe destacar, que el origen primario de los grupos es en la sociedad misma, la vía más adecuada para solucionar los problemas en grupo es estableciendo metas y direcciones comunes, donde todos trabajen hacia el mismo sentido, el mismo rumbo, sin menoscabo de la individualidad. 


\section{Zoila Rosa Montiel Florez}

De igual manera, considera, que la dinámica de grupo se refiere a la interacción constante e interna que mantienen los miembros de un grupo, lo cual es de carácter cambiante, dependiendo de las necesidades y situaciones particulares que se den en el mismo por cuanto los individuos son miembros de una familia, grupo religioso, comunidad, escuela, por tanto no se puede ver como un contexto aislado, al considerar que personas, sociedad son complementarias, no se desarrollan sino conjunta y dinámicamente en beneficio de la interacción entre los iguales.

Al respecto, Hung (2015), considera que las dinámicas de grupos se refiere a la interacción constante que mantienen miembros de un grupo, lo cual de un carácter cambiante, dependiendo de las necesidades y situaciones particulares que se den, igualmente las define como técnicas de discusión verbal, las cuales se pueden confrontar desde diferentes puntos de vistas, en un clima de armonía y de respeto permitiendo desarrollar la competencia comunicativa para ejercitar la pronunciación y practicar la coherencia entre tu tono de voz, gestos, el uso de un lenguaje adecuado, con las cuales las personas que las practique puede lograr tener unos mayores resultados en función de la práctica.

Así mismo, Motta (2014), refiere que el grupo no es una simple colección de individuos donde se producen múltiples fenómenos de: Atracción, repulsión, tensión, las corrientes establecidas entre los miembros, determinan un movimiento, una dinámica que proyecta en cierto modo, el grupo hacia delante, pero también puede haber un sentimiento de rechazo si esas impresiones no son compartidas, estas características unidas hacen que la dinámica del grupo se desarrolle en forma positiva o negativa.

\section{Tipos de Estrategias Lúdicas. Una respuesta a los escenarios educativos}

Las estrategias, de acuerdo a Viloria (2014), constituyen uno de los temas principales de la pedagogía. Las estrategias se refieren a los procedimientos necesarios para procesar la información, es decir, a la adquisición, a la codificación o almacenamiento y a la recuperación de lo aprendido, se vinculan a operaciones mentales con el fin de facilitar o adquirir un aprendizaje, a su vez que se entienden como todas aquellas acciones organizadas por el docente utilizando un conocimiento amplio al que presenta en el aula. 


\section{Zoila Rosa Montiel Florez}

De igual forma Guerrero (2014), refiere que diversas corrientes han subrayado su significación a partir de aproximaciones teóricas y metodológicas del más variado carácter para realizar su clasificación. En efecto, es sumamente difícil encontrar un área en la que no se planteen las condiciones que propician el surgimiento, la formación, el desarrollo y la evaluación de las mismas. Los tipos de estrategias son involucradas para comprender el espacio en el cual se desenvuelven los niños.

Por otro lado, Leal (2014), señala que la actividad lúdica es un ejercicio que proporciona alegría, placer, gozo y satisfacción. Es una dimensión del desarrollo humano que tiene una buena concepción porque no debe de incluirse solo en el tiempo libre, ni ser interpretado como juego únicamente. Lo lúdico es instructivo. El alumno, mediante lúdica, comienza a pensar y actuar en medio de una situación determinada que fue construida con semejanzas en la realidad. Con base a las teorías presentadas por los autores, puede referirse que los tipos de estrategias metodológicas puede apoyar los procesos de aprendizaje y modelar procesos didácticos alternativos a los tradicionales al generar motivación intelectual" y favorecer la relación entre maestro y alumno.

\section{Cuentos}

De acuerdo a Guerrero (2014), los cuentos surgen históricamente como relatos en prosa, de extensión variable, que tratan de personajes y hechos bien ficticios o de un pasado reconocible. Se componen siempre de una secuencia corta de motivos. En todo caso, le sirve al niño para dar vitalidad al espíritu, motivar la imaginación creadora, sensibilizarse, entre otros aspectos. Así mismo los cuentos estimulan el lenguaje comunicativo, a través de actividades significativas que ayudan en los procesos de aprendizaje.

De igual forma Delgado (2011), agrega que la primera estructura del cuento que un niño conoce es una composición muy sencilla que algunos autores denominan (precuento). Estas se suelen acompañar de gestos faciales y señalizaciones de partes del cuerpo. Algunas acompañan a rutinas cotidianas como comer, asearse o vestirse. Por ello el cuento que permite orientar el aprendizaje de la lectura en los niños; los cuentos tienen un enorme valor educativo por cuanto que: favorece el desarrollo del 


\section{Zoila Rosa Montiel Florez}

lenguaje, el vocabulario se amplía en el alumno (a) o de una forma sugestiva, a la vez proporciona modelos expresivos nuevos y originales, despiertan la afición por los textos escritos, estimulan la memoria y hacen evolucionar la estructuración temporal. También ayudan al desarrollo afectivo y social, en la medida en que el niño puede comprender el significado de los valores sociales y humanos históricamente relativos.

\section{Poemas}

En la poesía, se mezcla el ritmo y la rima e interesan al niño por la música con la que se dice. Señala, Guerrero (2014), que estas rimas se conocen también como precuentos, porque acercan al niño al mundo de los cuentos. Esto es así porque su estructura es sencilla, fácil de recordar, donde abundan las repeticiones, lo cual agrada al infante. Los poemas son una excelente herramienta didáctica para formar los valores que necesitan los niños para aprender a convivir en la sociedad.

Por otro lado, según Pérez (2011), desde siempre se ha visto la poesía como una herramienta, como un recurso que ayuda para motivar al alumnado cuando se abordan poesías que tienen relación con el tema que se esté tratando en clase. Esto es utilizar poesías que tengan que ver con los medios de transporte cuando se estén tratando en el aula, es decir, poner valor a poesías para motivar ante una temática concreta. La poesía acerca al entorno a través de los versos más básicos en los primeros años de escolarización.

Asimismo, para Díaz y Espeso (2010), los poemas se presentan para el estudiante como la gran oportunidad para manejar palabras, contemplarlas desde distintos ángulos y jugar con ellas. Muchas veces las palabras quedan a merced del niño con la exclusiva finalidad de crear un clima en el que pueda desarrollarse el juego, al que sirven de soporte o motivación las palabras. En la poesía infantil, sobre todo, el valor informativo pierde terreno ante el reclamo lúdico.

Es asi entonces, como los poemas son frases que riman y forman una estructura sencilla, para la enseñanza de los niños, es una herramienta que motiva a los alumnos y los acerca al mundo de los cuentos al mismo tiempo sirve para reforzar los valores. La poesía en la escuela ha de dar lugar al conocimiento del folclore popular y a la experimentación de la misma. 


\section{Zoila Rosa Montiel Florez}

\section{Juegos}

Según, Guerrero (2014), el juego se trata de una actividad natural del ser humano, en la que éste toma parte por la sola razón de divertirse y sentir placer. En la etapa escolar, cuando los estudiantes comparten, cooperan y disfrutan el acompañamiento de los otros, se fortalecen en ellos los sentimientos de pertenencia al grupo social con el que comparten; es así como se va solidificando su sentimiento de solidaridad. E juego es una estrategia que se puede utilizar en cualquier nivel o modalidad del sistema educativo, pero por lo general el docente lo utiliza muy poco.

Asimismo, Prieto (2010), dentro de las actividades recreativas, como estrategias indispensables, se aplica el juego y se considera como una acción improvisada; sin embargo, en los tiempos presentes, caracterizados por la presencia continua de cambios que abogan por la reforma educativa, el estudiante debe ser el centro del proceso educativo que como humano tiene una serie de expectativas, intereses, posturas, necesidades y metas en cada inicio de los lapsos escolares, a los cuales debe responder la escuela, con el fin de crear condiciones socio-laborales para realizar actividades y estrategias basadas en juegos intelectuales, como el debate, el ajedrez, entre otros.

Se igual forma para Sánchez (2008), el juego ofrece numerosas ventajas en el proceso de enseñanza-aprendizaje de una lengua. En él intervienen factores que aumentan la concentración del alumno en el contenido o la materia facilitando la adquisición de conocimientos y el desarrollo de habilidades. Entre sus grandes aportaciones podemos destacar que el juego es un componente lúdico importante para el proceso de enseñanza.

En ese sentido puede señalarse que el juego comprende una actividad recreativa, una acción libre, sentida como ficticia y situada al margen de la vida cotidiana, capaz sin embargo, de absorber totalmente al jugar, desarrollada en el nivel de educación inicial, esta se utiliza en el proceso de enseñanza-aprendizaje con la finalidad de motivar a los niños y aumentar su creatividad, los juegos se realizan por la sola razón de divertirse y sentir placer. 


\section{Zoila Rosa Montiel Florez}

\section{Canciones}

La canción, es otra herramienta lúdica que favorece a todos los aspectos del aprendizaje, dando prioridad a la destreza oral. Los descubrimientos musicales de acuerdo a Armijo (2013), estimulan los sentidos, el espíritu creativo y provoca la toma de consciencia del cuerpo en el movimiento musical, también se pueden asociar a esto el baile y el canto. La canción es también un documento autentico en la cual se pone en contacto el intercambio cultural.

Al respecto Guerrero (2014), expresa que, la canción es una forma de expresión musical en la que la voz humana desempeña el papel principal y tiene encomendada un texto; como término genérico, cualquier música que se cante; más específicamente, una composición vocal breve, sencilla, que consta de una melodía y un texto en verso. Presenta, por tanto, un texto, música y una relación entre ambos elementos. Escuchar, aprender y cantar canciones en clase es una estrategia lúdica práctica.

Finalmente, Palacios (2009), refiere que las canciones y sus contenidos han sido tradicionalmente herramientas educativas. Es más fácil encontrar el papel educacional en las canciones infantiles o en tradicionales o modernas como recurso en clases de un segundo idioma. A parte de los beneficios psicotónicos de este ejercicio, no hay duda que ellos nos permiten dejar contenidos en el área de segunda lengua en distintos niveles educativos. Además, como actividad lúdica, las canciones suponen una alternativa a otros ejercicios de repetición poco motivadores

Es por ello que, las canciones pueden ser definidas como una forma de expresión musical compuesta por versos y melodías sencillas acompañada de la voz humana, presentada por medio de, un texto, música y una relación entre ambos elementos, su contenido se conoce como una herramienta educativa importante en educación, las canciones son una forma de manifestar sentimientos difíciles de expresar, puesto que la música tiene un gran poder para la estimulación de las emociones. 


\section{Zoila Rosa Montiel Florez}

\section{METODOLOGÍA}

En este apartado se destaca la importancia de la metodología que se va a desarrollar en la investigación, considerando las características del paradigma que se ha seleccionado para tal fin. Es por ello que, desde el análisis de una investigación cualitativa, el desarrollo del estudio está en línea con el método descriptivo, del cual Hernández, Fernández y Baptista (2014), explican que son aquellos que "buscan especificar las propiedades, características y perfiles importantes de personas, grupos, comunidades o cualquier otro fenómeno que se someta a un análisis".

Del mismo modo indica Marin,Hernandez y Flores(2016) que bajo este enfoque, el investigador se encuentra en una interpretación permanente de acciones de los sujetos en estudio, dado que se busca comprender el contexto de su realidad.

En este sentido, de acuerdo a Tamayo y Tamayo (2012, p.46) manifiesta que la investigación descriptiva "trabaja sobre las realidades de hechos, y su característica fundamental es la de facilitar una interpretación del problema".

Asimismo, considerando el propósito que se persigue este tipo de estudio la técnica de la observación es la indicada, ya que permite inspeccionar ambientes, contextos, culturas y la mayoría de los aspectos de la vida social, así como las actividades que se desarrollan, en la búsqueda de comprender procesos, vinculaciones entre personas y sus situaciones, por último, identificar problemas y generar hipótesis para futuras investigaciones. En conjunto con la observación, la entrevista cualitativa, es la encargada de contribuir con este proceso, que es de carácter comunicativo gracias a la interacción de dos o más sujetos, por medio de la reconstrucción de los procesos, contenidos y vivencias experimentadas, está técnica es más flexible así como abierta e íntima.

\section{REFLEXIONES FINALES}

Las actividades lúdicas es un componente vital ya que esta manera los niños descubren cómo realizar juegos, lo que da lugar a una progresión de ejercicios divertidos y relajantes que son de primera calidad o más, pero que además han sido limitados. Sólo en condiciones específicas de ocasiones y lugares socialmente reconocidos, en este sentido, debe incorporarse dentro de los espacios de aprendizaje 


\section{Zoila Rosa Montiel Florez}

como un impulsor de esto, el uso de la acción energética como una técnica educativa para reforzar las aptitudes en el aprendizaje de los niños.

Se deben considerar varios puntos de vista en el docente que se encarga de alcanzar el desarrollo de las inteligencias múltiples con mayor calidad. El impacto de la sociedad y la cultura puede ser un impedimento para la perfecta ejecución del niño dentro del ambiente instructivo, así como también podría marcar su vida como un ser indispensable. Para investigaciones futuras identificadas con numerosos conocimientos, se prescribe utilizar varias dimensiones, tanto desde enfoques abiertos, donde se conectan modelos educativos.

La praxis educativa fusiona una situación en la evaluación de la educación, en la cual se considera importante relacionar la evaluación y sus medidas distintivas con la mejora experta del docente en un enfoque pre profesional, donde será importante crear criterios, instrumentos y metodologías de evaluación relacionados con las clases distintivas de información, ejecución y viabilidad expuestas en la planificación, y además de someterlo a un escrutinio serio en diversos entornos educativos y sociales.

\section{REFERENCIAS CONSULTADAS}

1. Alvarado, M. (2010). "El aprendizaje y la tutoría elementos clase del desempeño escolar de los universitarios". En:http://www.revistaeducarnos.com/art\%C3\%ADculos/educaci\%C3\%B3n/el aprendizaje-y-la-tutor\%C3\%AD-elementos-clave-del-desempe\%C3\%B10escolar-delos-universitarios.

2. Arce, A. C. (2015). Juegos educativos. Santiago: GRUPO INFAGON.

3. Dávila, J. (2013). El juego y la ludoteca. Talleres gráficos universitarios 1aㅡ impresión. Mérida: Venezuela.

4. Díaz y Espejo, L (2010). Poesía y educación poética. Cuenca:

5. Díaz-Aguado, M. (2006). Convivencia escolar y Prevención de la violencia. Madrid: Ministerio de Educación, Cultura y Deporte. 


\section{Zoila Rosa Montiel Florez}

6. Gallego, J.L. (2012): "Los contenidos en el proceso didácto", en A. Medina y F. Salvador (coords.): Didáctica general. Madrid: Prentice Hall.

7. Guerrero, R (2014) Estrategias lúdicas: herramienta de innovación en el desarrollo de las habilidades numéricas. Depósito Legal: PPI200802ZU2980 / ISSN: 1856-9331. Edición No 18. Año 9. Universidad Dr. Rafael Belloso Chacin. Venezuela.

8. Hernández, R., Fernández, C y Baptista, P. (2014). Metodología de la Investigación. México: Editorial Mc Graw Hill.

9. Huizinga, J (2012). Homo Ludens, Tercera edición, Editorial Alianza S.A., Madrid.

10. Hung, L (2015) "Modulo de Dinámica de Grupo". Universidad Nacional Abierta. Merida. Venezuela.

11. Jiménez, B. (2012) Lúdica y recreación. Bogotá: Editorial Magisterio.

12. Marin, A; Hernandez, E y Flores, J(2016) Metodología para el Análisis de datos Cualitativos en Investigaciones orientadas al aprovechamiento de fuentes renovables de energía. KOINONIA. Revista Arbitrada Interdisciplinaria de Ciencias de la Educación, Turismo, Ciencias Sociales y Económica, Ciencias del Agro y Mar y Ciencias Exactas y aplicadas. Año I. Vol I. №1. Ene - Julio, 2016.

13. Matos, C. (2012). Las inteligencias múltiples en estudiantes de tercer grado de secundaria de una Institución Educativa de Ventanilla-Callao. Lima: Universidad San Ignacio de Loyola.

14. Montenegro, L. (2010) El juego como actividad pedagógica para el niño de 12 meses a 5 años. Universidad Nacional Abierta. Caracas.

15. Motta, C. (2014). Fundamentos de la educación. Colombia: Cerlibre.

16. Núñez, J. (2010): "Ciencia, Tecnología y Sociedad", Problemas Sociales de la Ciencia y la Tecnología, GESOCYT, Editorial Félix Varela, La Habana.

17.Pacheco, G. (2015). Expresión Corporal en el Aula Infantil [Manual]. Recuperado

de http://www.runayupay.org/publicaciones/expresion corporal nivel inicial.pdf.

18. Pedagogía y Psicología Infantil (2010), Biblioteca Práctica para Padres y Educadores. La Infancia Edita: CULTURAL, S.A. Impreso en España. Madris España. 


\section{Zoila Rosa Montiel Florez}

19. Sánchez G. (2008) Comunicación efectiva en el aula. Técnicas de expresión oral para docentes. Barcelona: Graó.

20. Tamayo y Tamayo, M. (2012). El proceso de Investigación Científica. Editores Limusa.

21. Viloria, J. (2014). Estrategias aplicadas por los docentes promotores del aprendizaje significativo de las matemáticas en educación media general. Tesis de Grado. Maestría en Matemática, mención Docencia. Venezuela.

22.Zambrano, A (2007), "Tres tipos de saber del profesor y competencias: una relación compleja", Revista Educere, vol. 10, núm. 33, pp. 225-232.

23.Zielinski, T. (2010) Juegos y actividades preescolares. Ediciones Ceac. Barcelona. España. II Edición.

\section{REFERENCES CONSULTED}

1. Alvarado, M. (2010). "Learning and tutoring class elements of school performance of university students." In: http: //www.revistaeducarnos.com/art\%C3\%ADculos/educaci\%C3\%B3n/elaprendiz aje-y-la-tutor\%C3\%AD-elementos-clave-del-desempe\%C3\%B10-escolardelos-university.

2. Arce, A. C. (2015). Educational games. Santiago: INFAGON GROUP.

3. Dávila, J. (2013). The game and the playroom. University graphic workshops 1st impression. Mérida: Venezuela.

4. Díaz y Espejo, L (2010). Poetry and poetic education. Basin:

5. Díaz - Aguado, M. (2006). School life and violence prevention. Madrid: Ministry of Education, Culture and Sports.

6. Gallego, J.L. (2012): "The contents in the teaching process", in A. Medina and F. Salvador (coords.): General teaching. Madrid: Prentice Hall.

7. Guerrero, R (2014) Playful strategies: innovation tool in the development of numerical skills. Legal Deposit: PPI200802ZU2980 / ISSN: 1856-9331. Issue No. 18. Year 9. Dr. Rafael Belloso Chacin University. Venezuela.

8. Hernández, R., Fernández, C and Baptista, P. (2014). Investigation methodology. Mexico: Editorial Mc Graw Hill. 


\section{Zoila Rosa Montiel Florez}

9. Huizinga, J (2012). Homo Ludens, Third Edition, Editorial Alianza S.A., Madrid.

10. Hung, L (2015) "Group Dynamics Module". National Open University. Merida Venezuela.

11. Jiménez, B. (2012) Play and recreation. Bogotá: Magisterium Editorial.

12. Marine; Hernandez, E and Flores, J (2016) Methodology for the Analysis of Qualitative Data in Research oriented to the use of renewable energy sources. Koinonia Interdisciplinary Arbitrated Journal of Education, Tourism, Social and Economic Sciences, Agro and Sea Sciences and Exact and Applied Sciences. Year I. Vol I. No. 1. Jan - July, 2016.

13. Matos, C. (2012). Multiple intelligences in third grade high school students of a Ventanilla-Callao Educational Institution. Lima: San Ignacio de Loyola University.

14. Montenegro, L. (2010) Play as a pedagogical activity for the child from 12 months to 5 years. National Open University. Caracas.

15. Motta, C. (2014). Fundamentals of education. Colombia: Cerlibre.

16. Núñez, J. (2010): "Science, Technology and Society", Social Problems of Science and Technology, GESOCYT, Editorial Felix Varela, Havana.

17.Pacheco, G. (2015). Body Expression in the Children's Classroom [Manual]. Recovered from http://www.runayupay.org/publicaciones/expresion_corporal_nivel_inicial.pdf.

18. Pedagogy and Child Psychology (2010), Practical Library for Parents and Educators. La Infancia Edita: CULTURAL, S.A. Printed in Spain. Madris Spain.

19. Sánchez G. (2008) Effective communication in the classroom. Oral expression techniques for teachers. Barcelona: Graó.

20. Tamayo and Tamayo, M. (2012). The process of scientific research. Limusa editors.

21. Viloria, J. (2014). Strategies applied by teachers promoting significant learning of mathematics in general secondary education. Thesis. Master in Mathematics, mention Teaching. Venezuela.

22.Zambrano, A (2007), "Three types of teacher knowledge and skills: a complex relationship", Revista Educere, vol. 10, no. 33, pp. 225-232. 
CIENCIAMATRIA

Revista Interdisciplinaria de Humanidades, Educación, Ciencia y Tecnología

Año VI. Vol. VI. N¹. Edición Especial. 2020

Hecho el depósito de ley: pp201602FA4721

ISSN-L: 2542-3029; ISSN: 2610-802X

Universidad Nacional Experimental Francisco de Miranda (UNEFM). Santa Ana de Coro. Venezuela

Zoila Rosa Montiel Florez

23.Zielinski, T. (2010) Preschool games and activities. Ceac editions. Barcelona. Spain. II Edition.

2020 por los autores. Este artículo es de acceso abierto y distribuido según los términos y condiciones de la licencia Creative Commons Atribución-NoComercial-CompartirIgual 4.0 Internacional (CC BY-NC-SA 4.0)

(https://creativecommons.org/licenses/by-nc-sa/4.0/) 